\title{
Arbor
}

\section{La aceptabilidad de los alimentos: nutrición y placer}

Elvira Costell

Arbor CLXVIII, 661 (Enero 2001), 65-85 pp.

El proceso por el que el hombre acepta o rechaza un alimento tiene un carácter multidimensional con una estructura dinámica y variable. Considerando que la percepción humana es el resultado conjunto de la sensación que le hombre experimenta y de cómo él la interpreta, en este trabajo se comenta el papel de los principales factores que influyen en la aceptabilidad —el alimento, el hombre y su entorno-y se pone de manifiesto la necesidad de abordar su estudio desde una perspectiva multidisciplinaria.

\section{Introducción}

En el mundo animal, sólo aquellos organismos capaces de elegir los nutrientes que necesitan, en la proporción adecuada, tienen una evolución satisfactoria. Los seres humanos no son una excepción y necesitan ingerir una serie de compuestos para desarrollarse correctamente y para mantener una integridad física y funcional durante su vida. Aunque básicamente los animales tienden a seleccionar su dieta en función de sus necesidades nutritivas, en el caso del hombre la relación entre estas necesidades y el tipo y cantidad de los alimentos que consume no es tan obvia.

Con frecuencia, la selección e ingestión de los alimentos no se realiza teniendo en cuenta su contenido en carbohidratos, aminoácidos o vitaminas sino que las personas comen y beben determinados productos 
principalmente porque les gustan o les apetecen en un momento determinado. ¿Por qué una persona decide consumir un alimento? ¿por qué le gusta? ¿por qué le apetece? Es evidente que ni el contenido nutritivo ni las razones estrictamente hedónicas pueden justificar totalmente las tendencias de consumo ni los hábitos alimentarios de distintos grupos o poblaciones y menos todavía, los de los individuos que los integran. Las circunstancias personales por un lado y las culturales y sociales por otro, juegan un papel importante en la respuesta a las anteriores cuestiones.

Por ejemplo, la consideración de un determinado producto como alimento tiene un marcado carácter cultural y esto podría explicar porqué determinadas especies de animales (insectos, caracoles, ranas o perros), se consideran o no como productos comestibles en distintas culturas. Como comenta Fishler (1988), esta situación es difícil de explicar en función del contenido en nutrientes de estas especies; de hecho, muchos insectos son, por ejemplo, una buena fuente de proteínas. Tampoco se puede explicar tomando como base exclusivamente la sensación sensorial que sus componentes y propiedades físicas provocan en el consumidor ya que, en muchos casos, no existen grandes diferencias entre los de algunos de estos animales y los de otros que sí son consumidos habitualmente por una determinada población.

Otra cuestión es que los hábitos alimentarios de las distintas poblaciones han evolucionado y lo continúan haciendo, más o menos rápidamente, en función de los cambios sociales que se van produciendo en su entorno. A nivel histórico, los grandes cambios en los hábitos alimentarios asociados, por ejemplo, a la aparición de los animales domésticos, al desarrollo de las nuevas técnicas agrícolas o al de los procesos de industrialización de alimentos, han supuesto modificaciones importantes en la disponibilidad de alimentos lo que, indudablemente, ha repercutido en la aceptabilidad de los mismos y en el estado nutricional de los hombres en las diferentes épocas de la historia. Quizá en estos momentos estemos asistiendo al comienzo de una nueva etapa, en la que los alimentos obtenidos biotecnológicamente pueden llegar a ocupar un lugar importante. Pero, aparte de estos cambios necesariamente lentos, otras situaciones sociales, como las guerras o los periodos de hambre, pueden modificar rápidamente los hábitos alimentarios de la población. Cuando cesa la causa, estos hábitos vuelven a cambiar o no, según las circunstancias. Todo va a depender del grado de aceptación de las nuevas dietas o alimentos. Por ejemplo, el consumo de algunos productos (pan, legumbres o patatas) suele incrementarse en épocas de escasez, como la que sufrió Europa en la 
La aceptabilidad de los alimentos: nutrición y placer

Segunda Guerra Mundial, pero después, suele disminuir. Sin embargo, en algunos casos, un cambio en la dieta, provocado por una circunstancia especial, se traduce en una modificación importante de la misma que se mantiene posteriormente. Una ilustración de este hecho lo constituye la historia de la aceptación de la patata como alimento en Suiza (Ahebi, 1981). A pesar de que las primeras patatas llegaron a Europa en 1550 y que, ya en 1596, aparece la planta descrita en una publicación, tuvieron que pasar más de 200 años y producirse en el país una situación de hambre, para que fuera considerada como alimento humano. Hoy, nadie duda que muchos de los platos mas «tradicionales» de este país incluyen, como componente principal, este tubérculo.

Este planteamiento pone de manifiesto que el proceso por el que el hombre acepta o rechaza un alimento tiene un carácter multidimensional con una estructura dinámica y variable, no sólo entre los distintos individuos de un grupo sino incluso, para un mismo individuo en momentos y contextos diferentes. Por ello es complicado estudiar la aceptabilidad de los alimentos, intentar predecirla o interpretar su variaciones. Esta dificultad no impide que investigadores de distintas disciplinas estudien los diferentes aspectos del proceso por el que el hombre decide consumir determinados alimentos. En definitiva, todos estamos interesados en ello ya que nuestro estado nutricional y por tanto, nuestra salud y nuestra calidad de vida van a depender, en gran parte, de esta decisión.

\section{El proceso de aceptación de los alimentos}

Básicamente, la aceptación de los alimentos es el resultado de la interacción entre el alimento y el hombre en un momento determinado. Por un lado, las características del alimento (composición química y nutritiva, estructura y propiedades físicas) y por otro, las de cada consumidor (genéticas, etarias, estado fisiológico y sicológico) y las del entorno que le rodea (hábitos familiares y geográficos, religión, educación, moda, precio o conveniencia de uso), influyen en su actitud en el momento de aceptar o rechazar un alimento.

En la práctica, el principal problema que se presenta en el estudio del proceso que regula la elección e ingestión de los alimentos es que, en función del objetivo del estudio y de la especialidad de los autores, el planteamiento del problema suele tener un enfoque diferente y la metodología aplicada para registrar la respuesta humana, caracterís- 
ticas distintas. Por ello no siempre es fácil analizar comparativamente la información disponible.

Por ejemplo, en el área de la Tecnología de los Alimentos, el tema central suele ser el análisis de la relación entre la composición, estructura y propiedades del alimento y su aceptabilidad y los métodos más utilizados para investigar la opinión de las consumidores, suelen ser el registro de sus respuestas a cuestiones de tipo cuantitativo, generalmente en forma de escalas (Meiselman, 1994) aunque también se utilizan, en algunos casos, métodos de investigación cualitativos (Chambers y Smith, 1991). Sin embargo, en el área de la Neurofisiología de la Percepción, los temas centrales suelen estar relacionados principalmente con: a) la respuesta de los receptores frente a los distintos estímulos; b) las rutas neuronales y los mecanismos que gobiernan la transducción de la sensación percibida hasta el cerebro y c) el estudio de cómo reacciona éste. En este caso, la metodología aplicada puede oscilar entre el registro directo de las respuestas de los receptores (Brown, 1994) y el indirecto, por observación o medida de otras respuestas fisiológicas relacionadas con la percepción de un estímulo (Cowart y Beauchamp, 1990); o centrarse en el análisis de los cambios bioquímicos implicados en la transmisión del estímulo a través de las neuronas (Kurihara et al, 1989) o en la medida de la respuesta electroencefálica o de la actividad eléctrica cerebral que se registra como respuesta ante determinados estímulos (Van Toller, 1994). En el campo de la Sicofísica, los estudios se orientan a la modelización de la relación entre la magnitud del estímulo y la de la respuesta (Stevens, 1975; Norwich, 1991) aunque más recientemente se intenta desarrollar modelos sicofísicos integrados sobre la base de que la respuesta humana es casi siempre el resultado de la interacción entre diferentes estímulos (Mc Bride y Anderson, 1990). Existen muchas otras líneas de investigación como las orientadas a dilucidar el papel de las actitudes y de las creencias previas en la elección de los alimentos (Shepherd, 1990) o el de las expectativas sobre la calidad del producto generadas por sus características externas, marca o precio. (Cardello, 1994).

Varios autores han propuesto diferentes modelos teóricos para explicar el proceso de la aceptabilidad de los alimentos intentando incluir en los mismos los distintos factores que influyen y las conexiones e interacciones que pueden haber entre ellos. Desde que Pilgrim propuso su modelo en 1957 (Amerine et al, 1965) hasta el más reciente de Booth y Shepherd en 1988, ha habido distintas aportaciones de interés (Shepherd, 1989). Prácticamente en todas ellas se considera, más o menos implícitamente, que los factores que determinan la aceptabilidad 
La aceptabilidad de los alimentos: nutrición y placer

de un alimento responden a tres orígenes: el alimento, el hombre y el entorno. Las diferencias entre los distintos modelos residen, principalmente, en los factores que incluyen, en la importancia relativa que se les asigna y en la representación, mas o menos complicada, de los nexos que existen entre ellos.

De una forma simplificada, se puede considerar que la percepción que el hombre tiene de un alimento es el resultado conjunto de la sensación que éste le provoca y de cómo él la interpreta (Figura 1). La sensación que experimenta el hombre es la respuesta a los estímulos procedentes de los alimentos y el proceso de interpretación incluye referencias a informaciones o situaciones previas almacenadas en la memoria, que modulan la sensación percibida antes de decidir la aceptación o rechazo del alimento.

Figura 1. Esquema simplificado del proceso de aceptación de los alimentos

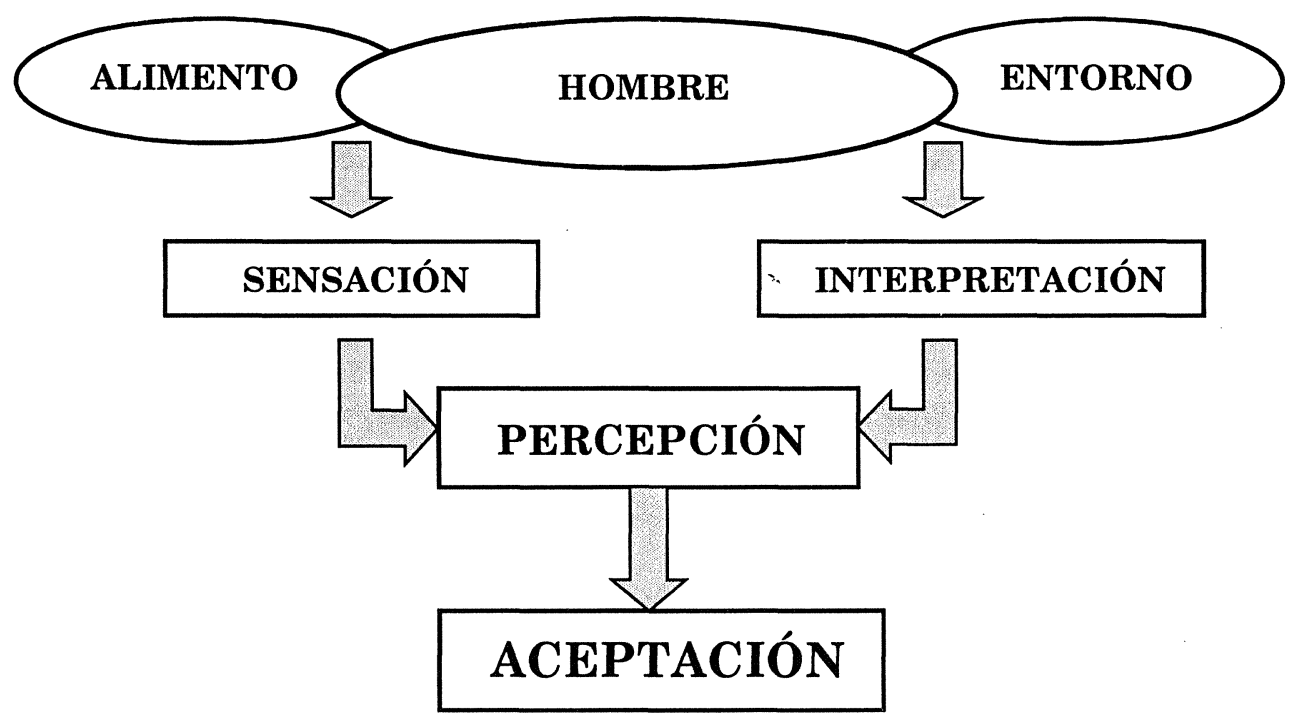

Factores que influyen en la sensación

La sensación que experimenta el hombre al observar e ingerir un alimento depende por un lado de la composición, propiedades físicas y características estructurales del producto y por otro, de su sensibilidad fisiológica, que le capacita para captar el estímulo procedente del alimento. 


\section{Los estimulos procedentes del alimento}

Las sensaciones sensoriales son siempre respuestas a estímulos externos y éstos son configuraciones de energía que, según su naturaleza, son capaces de activar un receptor sensorial u otro. De una forma general, la luz (energía electromagnética) activa el sistema visual; las vibraciones (energía mecánica) activan los sistemas auditivo y táctil y los compuestos químicos (energía química) activan los sentidos químicos, gusto y olfato (Guirao, 1981). Considerando los alimentos como origen del estímulo, sólo algunos de sus componentes o propiedades físicas son capaces de estimular los sentidos humanos y cuando lo hacen, la respuesta sólo se produce en un determinado intervalo de concentraciones o de magnitudes.

Quizá los primeros estímulos que se reciben de un alimento sean los visuales. De ellos recibimos información sobre el tamaño, forma y color de los alimentos y de estas sensaciones, las originadas por las propiedades ópticas del producto, tienen un especial interés. La respuesta del alimento a la luz que incide sobre él da lugar, principalmente, a la sensación que el hombre identifica como color y las características del mismo dependen fundamentalmente de la longitud de onda transmitida o reflejada por el alimento. Si consideramos, por un lado, la existencia del amplio espectro de frecuencias de energía electromagnética que conocemos y que somos capaces de medir instrumentalmente, por ejemplo, desde la de $10^{-14}$ metros, correspondiente a los rayos gamma, hasta la de $10^{5}$ metros, que nos permite oír la radio, y observamos, por otro, que sólo una estrecha banda de las mismas, la comprendida entre 400 y $700 \mathrm{~nm}$, es capaz de estimular el ojo humano y además que, dentro de ella, pequeñas variaciones de longitud de onda provocan sensaciones cualitativamente distintas, tendremos una idea sobre la diferencia que puede haber entre el mundo físico y el que somos capaces de detectar sensorialmente.

Una relación parecida se puede establecer entre las propiedades mecánicas de los alimentos, que son las que se ponen de manifiesto cuando actúan sobre ellos fuerzas externas, y las sensaciones de viscosidad y textura. Aunque en éstas influyen también los sentidos de la vista y el oído, es el sentido del tacto y la respuesta de los receptores musculares y de las articulaciones los que definen la ruta capaz de traducir una fuerza o deformación mecánica a un código neuronal. La relación entre el estímulo y la respuesta en este caso no está tan clara como en el del color y es difícil establecer qué propiedad o propiedades mecánicas del alimento son los estímulos que dan lugar, aunque 
sea parcialmente, a las sensaciones percibidas. Mientras que en los alimentos líquidos de flujo newtoniano, en los que la viscosidad es independiente de la fuerza aplicada (agua, vino, aceite) parece que el estímulo de la viscosidad que se percibe sensorialmente es la viscosidad instrumental, en los que fluyen con carácter no-newtoniano (concentrados de frutas, purés, sopas) la naturaleza del estímulo no está bien identificada (Costell y Durán, 2000). En el caso de la textura de los alimentos sólidos el problema se complica y a pesar de los avances conseguidos (Brennan, 1988) sólo algunas de las propiedades mecánicas como la resistencia a la deformación, a la rotura, al corte, etc parecen estar relacionadas claramente con algunas de las sensaciones percibidas cuando se manipula y mastica el alimento.

El gusto y el olfato se suelen denominar «sentidos químicos» porque responden a estímulos que son esencialmente grupos atómicos o moléculas capaces de activar los receptores específicos situados en la boca y en la cavidad nasal. Los estímulos del gusto son compuestos químicos no volátiles y solubles en agua y los del olfato, son sustancias volátiles. La percepción conjunta de ambos conforman lo que se conoce popularmente como sabor. Existen otros estímulos, que también se perciben en la cavidad bucal y que se transmiten por el nervio trigémino, que suelen considerarse como sensaciones irritantes (picante, ardiente, mentolado, refrescante, etc).

Existe un elevado número de compuestos químicos en los alimentos (mono y disacáridos, polialcoholes, terpenos, ureas, péptidos, alcaloides, procianidinas, ácidos, sales, etc) capaces de estimular las papilas gustativas. Sin embargo, tradicionalmente, se ha considerado que sólo pueden producir cuatro tipos de sensaciones diferentes o fundamentales: Dulce, amargo, ácido y salado. Actualmente se acepta una quinta sensación como fundamental y diferente de las anteriores, el umami (Kawamura y Kare, 1987). Este gusto, identificado inicialmente con la sensación provocada por el glutamato monosódico, se consideró en principio como un modificador de los gustos primarios porque pequeñas cantidades del mismo, añadidas a algunos alimentos, los hacía mas sabrosos. Sin embargo parece que su adición no modifica directamente la percepción de los gustos fundamentales y por tanto, que no los refuerza directamente sino que su efecto se produce a través de un proceso sinérgico con los nucleótidos contenidos en diferentes alimentos (Kurihara, 1987). Algunos autores han sugerido otros gustos como fundamentales, como el metálico o el alcalino pero no existe evidencia sobre la naturaleza de los estímulos que los provocan ni sobre su mecanismo de percepción. De hecho, en función de los diferentes me- 
canismos receptores de los diferentes gustos, el concepto de gusto fundamental o primario, a pesar de su utilidad práctica, no tiene hoy un significado absoluto (Kurihara, 1987; Delwiche,1996). A pesar de los avances producidos en el estudio de los mecanismos que regulan los procesos de quimiorecepción (Durán y Costell, 1999), es difícil predecir, en función de su composición o estructura química, si un determinado compuesto del alimento va a ser capaz de estimular las papilas gustativas y si lo hace, qué tipo de sensación va a provocar. Es bien conocido que pequeñas diferencias en la composición química o en la configuración estérica de algunas moléculas dan lugar a sensaciones gustativas distintas (Amerine et al, 1965). Por ejemplo, cuando se sustituye el azufre de la molécula de feniltiocarbamato, que da sabor amargo, por un oxígeno se obtiene el compuesto conocido por dulcina que es, aproximadamente, 300 veces mas dulce que la sacarosa. En cuanto a la influencia de la configuración estérica, muchos aminoácidos como la leucina, isoleucina, valina, histidina o triptófano se perciben como dulces en su forma dextro pero no en su forma levo y la isomaltosa origina una sensación dulce mientras que su anómero, la gentibiosa, se percibe como amarga.

El aroma de los alimentos está ligado a la presencia de un elevado número de compuestos volátiles muchos de los cuales es lógico pensar que no se hayan identificado todavía. Pero de los cientos de compuestos volátiles identificados en diferentes tipos de alimentos y bebidas (Tabla 1) sólo un porcentaje relativamente bajo de ellos, en unas concentraciones determinadas y en unas proporciones específicas, dan lugar al olor o aroma característico de algunos productos. En ocasiones, sólo uno de ellos es el principal responsable de la sensación experimentada por el hombre. Por ejemplo, de los 51 componentes encontrados en el aceite esencial del anís, uno de ellos, el anetol, es el principal responsable de su aroma (Boelens,1991). Si bien, conforme se incrementa la investigación en este campo, se obtiene información que permite evaluar la contribución de determinados componentes minoritarios que pueden modificar cualitativamente la sensación percibida. El caso del aroma del limón puede ilustrar esta situación. Tradicionalmente se ha considerado que el citral, que es un aldehído mezcla de dos isómeros, el neral y el geranial, era el responsable del aroma de limón. Pero hay datos de otros componentes como el $\beta$-pineno, el $\gamma$-terpineno, el geraniol y el cariofileno, entre otros, que modifican el olor propio del citral (Shaw, 1991) y contribuyen al aroma que es identificado como «a limón» por el hombre. Si en el caso de los compuestos químicos capaces de producir sensaciones gustativas era difícil establecer rela- 
ciones generales entre su composición y estructura y el gusto, en el caso de los olores el problema es mayor. Productos de composición química muy similar pueden dar lugar a olores muy diferentes mientras que sustancias de distinta composición, si tienen una forma y un tamaño molecular parecidos, pueden originar olores similares. Como comenta Lawless (1991), es fácil distinguir entre el aroma de la lavanda y el del orégano y sin embargo, ambos tienen un perfil de volátiles muy similar y ni la identificación y cuantificación de los picos de sus cromatogramas explica claramente a qué se deben las diferencias detectadas entre el aroma de ambos compuestos. Otro problema adicional cuando se identifica, de forma errónea, el cromatograma de una sustancia con su aroma, es que algunos compuestos tienen un fuerte impacto sensorial a concentraciones muy bajas mientras que otros volátiles, presentes en altas concentraciones, tienen poco o nulo impacto sensorial.

TABLA 1. Número de compuestos volátiles identificados en algunos tipos de alimentos y bebidas

\begin{tabular}{||l|c|l|c||}
\hline \multicolumn{1}{|c|}{ Producto } & N. $^{0}$ de volátiles & \multicolumn{1}{c|}{ Producto } & N..$^{\circ}$ de volátiles \\
\hline Manzana & 356 & Endivia & 119 \\
\hline Cerveza & 562 & Uva & 466 \\
\hline Mantequilla & 257 & Naranja & 203 \\
\hline Cacao & 503 & Tomate & 385 \\
\hline Café & 790 & Vino blanco & 644 \\
\hline
\end{tabular}

Fuente: Maarse, 1991.

Los alimentos, por tanto, no tienen color, ni textura, ni gusto, sabor o aroma, sino unas determinadas propiedades físicas o estructurales y unos componentes químicos capaces de estimular los sentidos humanos. Para que estos estímulos sean percibidos por el hombre es necesario además, que estén en unas determinadas concentraciones o sean de una magnitud concreta.

\section{La relación entre el estímulo y la respuesta}

Igual que ocurre con el estímulo visual, que sólo un pequeño intervalo de longitudes de onda puede ser captado por el ojo humano, sucede con los otros sentidos. Es necesario que el estímulo alcance una de- 
terminada magnitud para ser percibido por el hombre y una magnitud ligeramente superior para ser reconocido. La primera es la que se conoce como umbral de detección y marca el momento en que se inicia la relación entre el estímulo físico y la respuesta humana, la segunda, recibe el nombre de umbral de reconocimiento. Es evidente que existe una gran variabilidad entre los umbrales de detección de diferentes personas e incluso, entre los de una misma persona, en función del momento y de la situación en que se encuentre. Por ello, en la práctica, se considera como valores umbrales de un determinado estímulo aquel que es capaz de ser percibido o reconocido por el $50 \%$ de la población (Tabla 2). Como comentaremos mas adelante, esta solución puede resultar adecuada cuando los umbrales individuales tienen una distribución normal, pero puede presentar problemas de interpretación en los casos en que dicha distribución se aleja de la normalidad o incluso, es claramente bi- o multimodal.

TABla 2. Concentraciones de diferentes estímulos, en disoluciones acuosas, necesarias para que el $50 \%$ de la población reconozca los distintos grupos

\begin{tabular}{||l|l|c||}
\hline \multicolumn{1}{|c|}{ Gusto } & \multicolumn{1}{|c|}{ Estímulo } & $\begin{array}{c}\text { Concentración } \\
\text { g/L }\end{array}$ \\
\hline Ácido & Ácido cítrico (monohidratado) & 0,43 \\
\hline Amargo & Cafeína cristalizada (monohidratada) & 0,195 \\
\hline Salado & Cloruro de sodio (anhidro) & 1,19 \\
\hline Dulce & Sacarosa & 5,76 \\
\hline Umami & Glutamato de sodio (monohidratado) & 0,595 \\
\hline
\end{tabular}

Fuente: UNE: 87003 (1995).

Una vez establecida esta relación, es de esperar que si se modifica la magnitud del estímulo se produzca una variación en la sensación. Si esta variación es cuantitativa, como por ejemplo cuando se añade azúcar a un zumo de frutas y se nota un incremento de dulzor, estamos ante lo que se conoce en sicofísica como un continuo protético. Si la variación del estímulo da lugar a una modificación cualitativa en la sensación, como ocurre con los diferentes colores que vemos cuando se modifica la longitud de onda que percibimos, entonces estamos ante un continuo metatético (Stevens, 1975). Estos dos tipos de continuos parece que obedecen a principios de percepción fisiológica distintos. En el caso del protético, la modificación de la intensidad de la sensación correspondería a un mecanismo aditivo y en los continuos metatéticos 


\section{La aceptabilidad de los alimentos: nutrición y placer}

estaríamos ante mecanismos sustitutivos en los que un receptor deja de ser activado a favor de otro (Guirao, 1981).

En el estudio de la relación entre el estímulo y la respuesta existen otros dos temas de especial interés, sobre todo en los continuos protéticos. Uno está relacionado con cuánto se tiene que incrementar el estímulo en cada caso para dar lugar a un incremento en la sensación y esta magnitud es la que se conoce como umbral diferencial. El otro, está relacionado con las leyes que expresan las relaciones funcionales existentes entre la variación de la magnitud del estímulo y la variación de la magnitud de la sensación. Entre las leyes sicofícas clásicas, la ley de Stevens es una de las mas populares y establece que «a proporciones iguales del aumento del estímulo corresponden proporciones iguales del aumento de la sensación» o dicho de otra forma, la relación entre la magnitud de la sensación (S) y la del estímulo (I) es de tipo potencial: $\mathrm{S}=\mathrm{kI}^{\mathrm{n}}$. El valor del exponente $n$, define el factor de proporcionalidad entre el estímulo y la respuesta y da una idea sobre la capacidad de discriminación de un sistema sensorial. Cuando el valor de $n$ es inferior a la unidad, indica que es necesario un incremento relativo mayor de la intensidad del estímulo para lograr un incremento de la sensación. Cuando el valor de $n$ es superior a la unidad, ocurre lo contrario, pequeños incrementos del estímulo dan lugar a incrementos detectables de la sensación. En la tabla 3 se recogen algunos de los valores del exponente $n$ de interés en el campo de los alimentos. Si se comparan, por ejemplo, los valores de los exponentes obtenidos para el sabor dulce de la sacarosa $(n=1,30)$ y el de la sacarina $(n=0,80)$ podemos concluir que hace falta un incremento relativo mayor en la concentración de sacarina que en la de sacarosa para incrementar la intensidad del sabor dulce que se percibe.

TABLA 3. Relación entre la magnitud del estímulo y la magnitud de la sensación. Valores del exponente $n$, correspondiente a la ley de Stevens $\left(\mathrm{S}=\mathrm{kI}^{\mathrm{n}}\right)$

\begin{tabular}{||l|c|l||}
\hline \multicolumn{1}{|c|}{ Continuo } & Exponente & \multicolumn{1}{c|}{ Estímulo } \\
\hline Olfato & 0,55 & Café \\
Olfato & 0,60 & Heptano \\
Gusto & 0,80 & Sacarina \\
Gusto & 1,30 & Sacarosa \\
Gusto & 1,30 & Cloruro sódico \\
Viscosidad & 0,45 & Silicona fluida \\
Dureza táctil & 0,80 & Compresión de goma entre los dedos \\
\hline
\end{tabular}

Adaptado de Guirao (1981) 


\section{La respuesta humana}

La respuesta humana a los estímulos procedentes de los alimentos es muy variable y está condicionada por diversos factores de distinta naturaleza. Unos, dependientes de las características particulares de cada individuo y otros, relacionados con sus condiciones fisiológicas y sicológicas en el momento de establecer el contacto con el alimento. No es el objetivo de este trabajo hacer una enumeración exhaustiva de todos y cada uno de los factores que influyen en la respuesta humana sino comentar algunos de especial interés.

Entre las características propias de cada individuo, las genéticas (incluido el sexo), la edad y la personalidad son las que han recibido una mayor atención. Entre las que pueden ejercer una influencia puntual, las de tipo fisiológico (hambre, saciedad, sueño, salud, adaptación) son las que se considera que más pueden influir en la magnitud de la respuesta, en un momento determinado.

La contribución de las características genéticas y del sexo a la variabilidad de sensibilidad entre las personas que tienen los sentidos del gusto y del olfato «normales» no está bien definida. Si bien algunos autores han detectado una mayor sensibilidad al dulce y al salado en las mujeres y una mayor sensibilidad al ácido en los hombres (Amerine et al 1965) los datos no han sido suficientemente confirmados ni explicados satisfactoriamente. Parece que otras causas, como la personalidad, la salud o determinadas condiciones fisiológicas hacen que se detecten mayores diferencias entre personas de un mismo sexo que cuando se compara la sensibilidad de ambos. Quizá una de las causas de esta situación sea que, excepto en algunos casos, no son muy frecuentes los estudios genéticos del proceso que regula la quimiorecepción en los mamíferos (Wysocki y Beauchamp, 1991). La mayoría de los estudios en este campo se han centrado en el análisis de los comportamientos que se alejan del considerado como «normal» como vía para llegar a dilucidar el mecanismo que regula la percepción sensorial de los distintos estímulos. Por la importancia de la visión y la clara identificación de los estímulos responsables de las sensaciones que se traducen como colores, las anomalías permanentes en la percepción de los mismos son las mas investigadas. Estas anomalías, que popularmente se denominan de forma genérica como daltonismo, responden a una alteración genética ligada al cromosoma $\mathrm{X}$, de carácter recesivo, que se manifiesta por la falta de alguno de los pigmentos sensibles al color, que habitualmente se encuentran en los conos de la retina y se traduce en la ceguera a determinados colores. La mayor parte 
La aceptabilidad de los alimentos: nutrición y placer

de las personas con esta anomalía, no son capaces de ver el verde (deutanopes), otra parte tiene problemas para ver el rojo (protanopes) y sólo un pequeño porcentaje es incapaz de ver el azul (tritanopes). En este caso, la alteración sí aparece ligada al sexo y es más frecuente en los hombres, que la presentan en un porcentaje del $8 \%$, que en las mujeres en las que el porcentaje de aparición de la ceguera a los colores no llega al 0.5\% (Lawless y Heymann, 1998).

En el caso del gusto y del olfato también se detectan alteraciones permanentes en personas que, con una sensibilidad normal frente a la mayoría de los gustos y de los olores, son incapaces de percibir determinados estímulos. Estas «cegueras» ante determinados compuestos se describen como ageusias (gusto) y anosmias (olor) específicas. Entre las primeras, uno de los ejemplos más conocidos es la insensibilidad ante los compuestos que contienen el grupo funcional $\mathrm{N}-\mathrm{C}=\mathrm{S}$ que, habitualmente, tienen sabor amargo. Las primeras noticias de esta ageusia, datan de 1930, cuando Fox (Lawless, 1991) detectó que determinadas sustancias eran amargas para muchas personas y totalmente insípidas para otras y que la distribución de los datos obtenidos al analizar la relación entre la intensidad del estímulo y su percepción era claramente bimodal. Los estudios iniciales se realizaron con la feniltiourea (PTC) aunque posteriormente, se sustituyó por el propiltiouracil (PROP) que es menos tóxico y no tiene olor sulfuroso. El interés de esta anomalía reside en que parece que la presenta un elevado porcentaje de personas de la raza blanca caucasiana $(\sim 35 \%)$ mientras que no se ha detectado en grupos africanos ni orientales. Genéticamente parece que se trasmite siguiendo la leyes de la herencia mendelianas y que requiere la presencia de dos alelos recesivos. El interés por este caso se ha incrementado recientemente al ponerse de manifiesto que, además de la gente incapaz de detectar el gusto amargo de estas sustancias, entre los que lo detectan, existe un grupo hipersensible. Hasta qué punto los que no lo detectan pueden percibir otros gustos del alimento como más intensos y los hipersensibles pueden percibir determinados alimentos, como ciertas frutas y verduras, con un excesivo sabor amargo, es un tema de interés actual por su posible implicación en la nutrición. Ya en 1954, Fox consideró la posible transcendencia de esta situación y la puso de manifiesto en la conferencia que pronunció en la reunión de la American Chemical Society con el título: Why Johnny likes spinach and Mary doesnt? (Lawless, 1991).

El concepto de anosmia específica fue introducido por Amoore en 1967 y desde entonces, el mismo autor ha descrito mas de 76 ejemplos de anosmias a diferentes compuestos químicos (Amoore y Steinle, 1991) 


\section{Elvira Costell}

que afectan a un mayor o menor porcentaje de la población (tabla 4). Según estos autores, la existencia de estas anosmias implica que el sistema olfativo se apoya en sensores químicos específicos y que podrían explicarse por la falta o malformación de determinadas proteínas receptoras. Es lógico pensar que los genes jueguen un papel importante en este proceso pero es difícil actualmente concluir los orígenes de la herencia de estas alteraciones de la respuesta humana. Por ejemplo, la anosmia a la androsterona, compuesto responsable de un olor desagadable en muchos productos derivados del cerdo, parece que tiene una fuerte base genética pero la influencia en la evolución de la sensibilidad a este compuesto con la edad - rara vez los niños presentan esta anosmia y sin embargo, el porcentaje de anósmicos se incrementa después de la pubertad- y la que se registra con la exposición continuada al estímulo, obliga a matizar esta conclusión (Wisocky y Beauchamp, 1991). Otras anosmias interesantes por su relación con los alimentos son la del aroma a malta, provocado por el isobutiraldehído y la del aroma a rancio, por la trimetilamina. La primera hace que una parte de la población sea poco sensible a la alteración del sabor y aroma de la leche contaminada por el Streptococcus lactis v. maltigenes y que otra, lo sea ante el deterioro de los pescados con el almacenamiento. Pero, aparte de la incidencia directa de estas alteraciones en la percepción de estímulos concretos, hay que tener en cuenta su influencia en las características cualitativas percibidas en el aroma de determinados alimentos como consecuencia de dejar de percibir uno de sus componentes (O־Conell, 1991).

TABla 4. Algunos de los compuestos para los que se ha detectado anosmias específicas y porcentaje de adultos anósmicos para cada uno de ellos

\begin{tabular}{||l|c|l|c||}
\hline \multicolumn{1}{|c|}{ Compuesto } & Anósmicos (\%) & \multicolumn{1}{|c|}{ Compuesto } & Anósmicos (\%) \\
\hline Androsterona & 47 & 1-Carvona & 8 \\
\hline Isobutiraldehído & 36 & Trimetilamina & 6 \\
\hline 1-Pirrolina & 16 & Ácido isovalérico & 3 \\
\hline
\end{tabular}

Fuente: Wysocki y Beauchamp, 1991.

Pero no sólo estos tipos de anomalías regulan la respuesta del hombre frente a los distintos estímulos. Otros factores, como la edad, juegan un papel importante en la sensibilidad. En los niños recién nacidos, parece que existe una reacción clara a los estímulos dulce y 
ácido pero su reacción ante los estímulos amargo y salado es más dudosa. Existe poca información y no siempre está contrastada, sobre la evolución de la sensibilidad en el periodo posterior (1-24 meses) debido, en parte, a la dificultad de medir la sensibilidad en niños de esta edad (Cowart y Beauchamp, 1990). Sin embargo, sí está demostrado que existe un moderado incremento en los umbrales de percepción de los distintos estímulos químicos al aumentar la edad, si bien este efecto es más importante en los aromas que en los gustos. La disminución del número de papilas gustativas con la edad, ha sugerido una pérdida de la sensibilidad del gusto en las personas mayores que no se corresponde con los resultados experimentales. La disminución de la sensibilidad a los diferentes gustos no es muy acusada y además es específica para cada uno de ellos; la mayor pérdida se produce en el amargo y la menor, en el dulce. Ello indica que en el envejecimiento del sentido del gusto influye más la modificación de la membrana y del mecanismo receptor que la disminución del número de papilas gustativas. En el caso de la sensibilidad a los aromas, la edad sí que juega un papel importante y se ha comprobado una clara disminución de la misma que también se produce en las sensaciones trigeminales. Por ello, muchos de los componentes responsables del aroma y del sabor de los alimentos dejan de percibirse lo que puede afectar a su aceptabilidad e incidir en la selección de la dieta y por tanto, afectar al contenido nutritivo de la misma (Murphy y Gilmore, 1990).

Entre los factores de carácter no estrictamente fisiológico, la personalidad, es una de las características del hombre relacionada con su sensibilidad a diferentes estímulos. Utilizando las respuestas a la prueba PBC (Private Body Conciousness) las personas se clasifican como de alta o baja consciencia frente a los cambios que se producen en su cuerpo. Existen datos que avalan una mayor sensibilidad a los diferentes gustos y a algunas sensaciones trigeminales en las personas de alto PBC (Stevens,1991). En una experiencia realizada sobre la variación de la intensidad del sabor salado percibido en una sopa de pollo al incrementar la concentración de sal, al estudiar la relación entre el estímulo y la respuesta se observó que para el grupo de personas con alto PBC, el exponente $n$ de la ley de Stevens tenia un valor de 1,168 mientras que, para el grupo de bajo PBC, el valor de $n$ era de 0,846. (Stevens et al, 1988). En el análisis de la relación entre la personalidad y la sensibilidad a los diferentes estímulos la cuestión es ¿las diferencias en personalidad dan lugar a personas de distinta sensibilidad o las personas con distinta sensibilidad a los estímulos externos desarrollan personalidades de rasgos diferentes? 
La influencia de todos estos factores y de otros no comentados en este trabajo, como los relacionados con el hambre, el sueño, algunas enfermedades, etc. determinan la magnitud de la respuesta humana a los estímulos procedentes del alimento pero no la aceptabilidad de los mismos. No todos los estímulos que se perciben influyen en la aceptabilidad, ni tampoco lo hacen todas las diferencias de magnitud detectadas en los mismos. Depende de la interpretación personal de las sensaciones experimentadas el que éstas se traduzcan en la aceptación o no, de un determinado producto. Como ha comentado recientemente el Prof. Mira (1999), «estudios neurofisiológicos han puesto de manifiesto que las neuronas no responden a ningún componente hedónico asociado al estímulo. El cerebro procesa inicialmente la información sensorial de una manera desprovista de todo componente emocional y sólo cuando el estímulo alcanza ciertas áreas, los llamados «circuitos límbicos» es cuando adquiere la tonalidad afectiva y emocional».

\section{Factores que influyen en la interpretación de la sensación}

En una primera aproximación, la aceptación del alimento durante la infancia está regulada por las necesidades fisiológicas del niño y responde a determinados mecanismos neurofisiológicos y bioquímicos que regulan la sensación de apetito. Los niños recién nacidos tienen una preferencia innata por el dulce y también, una aversión por el ácido. La respuesta al amargo está menos clara, porque muestran disgusto frente a la quinina pero no, frente a la urea. Parece que son bastante insensibles al gusto salado aunque ya a los cuatro meses, se ha detectado una preferencia en la ingestión de agua con sal al compararla con la ingestión de agua sola (Cowart y Beauchamp, 1990). Sin embargo, no está claro que existan respuestas innatas de aceptación o de rechazo a diferentes olores, colores o texturas. Lo que sí es cierto es que el niño aprende en poco tiempo a conectar la sensación de placer o de aversión con el gusto de los alimentos y éste con su aspecto y su textura y empieza a desarrollar sus preferencias. Este aprendizaje se desarrolla en función de sus propias experiencias y esta relacionado con su dieta, que le hace familiarizarse con determinado tipo de alimentos, pero también, está claramente influido por los hábitos alimentarios de su familia y, en algunos casos, por reacciones emocionales derivadas de su relación con sus padres o cuidadores. Las preferencias y aversiones desarrolladas durante la infancia, que influyen en los productos que consumen y por tanto, en su estado nutricional, se van 
modificando con la edad por la incidencia en las mismas de las condiciones particulares de cada consumidor y por las condiciones sociales y culturales del entorno que le rodea.

Considerando el entorno que rodea al hombre, la disponibilidad de los alimentos, los hábitos alimentarios de la sociedad en que vive, su nivel cultural, su conocimiento sobre las características y el contenido nutritivo de los alimentos, sus creencias religiosas, las modas o el precio, van modificando paulatinamente los criterios de preferencia de los seres humanos a lo largo de su vida. Sólo así se explica, por ejemplo, que una aversión inicial por el gusto amargo se transforme no sólo en una característica aceptable sino incluso, deseable, en productos de elevado consumo como el café o la cerveza. Algo similar ocurre con otras sensaciones como las ligeramente irritantes de las bebidas carbónicas o las picantes propias de determinadas especias. En estos casos, la moda y las costumbres sociales juegan, aparentemente, un papel decisivo. Sin embargo, si consideramos el efecto fisiológico estimulante del café y del alcohol o las inmediatas respuestas fisiológicas a la ingestión de sustancias picantes (incremento de la salivación que mejora la transmisión del sabor de otros productos, secreción de endomorfinas) se debe matizar hasta qué punto ciertos factores externos pueden llegar a modificar determinados hábitos. Lo mismo ocurre con la información acerca de los posibles efectos que determinadas sustancias pueden tener en nuestra salud. ¿Influyen en nuestra elección de la dieta? Otra vez la respuesta es ambivalente: sí y no. Depende en cada caso de la relación personal que se establece entre el placer de consumir determinado alimento y el daño que nos puede causar. Si éste es inmediato o importante (alergias, náuseas) suele ser suficiente para rechazar el alimento pero si el daño aparece como más difuso y lejano (elevación del nivel de colesterol, enfermedades futuras, etc.) su posible influencia en la dieta es menor porque el placer de comer es inmediato mientras que las posibles consecuencias negativas se esperan a largo plazo.

Cuando el cerebro del hombre procesa conjuntamente la información procedente de las sensaciones que experimenta al observar, manipular y consumir el alimento, la información adquirida del contexto social y cultural que le rodea y la obtenida de los efectos fisiológicos (placer, saciedad, desagrado, malestar, etc.) que experimenta al ingerir y después de ingerir un determinado alimento y las compara con la información almacenada en la memoria de experiencias anteriores, se produce la aceptación o el rechazo del alimento. Cualquier variación temporal en alguno de los factores que contribuyen a ello puede y de hecho lo 
hace, modificar el sentido de la respuesta. Esta situación obliga, para entender e interpretar el proceso por el que el hombre acepta o rechaza un alimento, a enfocar el problema desde un punto de vista multidisciplinario y a interpretar con mucha prudencia, los resultados obtenidos al estudiar la relación entre unos determinados factores y la aceptabilidad de un alimento.

En este sentido, hay que tener en cuenta que cuando, en una investigación, los orígenes de las diferencias individuales no se identifican, la variabilidad detectada en los datos experimentales se incluye en el término del error al analizarlos estadísticamente. En la mayoría de los trabajos que se realizan sobre este tema, el tratamiento estadístico de los datos suele apoyarse en una suposición que no siempre responde a la realidad: Se considera que los consumidores de una determinada edad o los pertenecientes a un determinado grupo étnico o a una determinada categoría social, reaccionan frente a los alimentos de acuerdo con un patrón de comportamiento común y que de los datos agrupados según criterios de este tipo, se puede inferir cómo va a reaccionar o lo que va a preferir cualquier individuo del grupo o por lo menos, la mayoría de los individuos que lo integran. En realidad, las relaciones entre los diferentes factores que determinan la elección e ingestión de un alimento en un momento determinado es, como hemos comentado, una cuestión totalmente individual y el uso de un valor medio, obtenido de un grupo del que se desconoce la variabilidad y el origen de la misma, puede dar resultados totalmente erróneos (Booth, 1990). El estudio previo de la estructura de los datos experimentales, mediante algunas técnicas estadísticas multivariantes como la de los conglomerados o la de los mapas de preferencia internos (Williams,1988; Greenhoff y MacFie, 1994) puede ayudar a detectar si dentro de un grupo de personas de características fisiológicas, étnicas o sociológicas comunes, existen subgrupos con patrones de comportamiento o con criterios de preferencia distintos y si esto ocurre, al analizar por separado los datos obtenidos para cada uno de estos subgrupos, se puede obtener una información más representativa de las pautas de comportamiento o de las preferencias reales del grupo estudiado (Damásio et al, 1999). Idealmente, el poder identificar las razones que explican los diferentes criterios de aceptabilidad de los distintos grupos, aportaría una información interesante para el diseño de dietas y para la formulación de nuevos alimentos, especialmente los destinados a grupos con unos requerimientos nutritivos especiales.

En última instancia, sólo los alimentos que se consumen con placer tienen una incidencia importante en la nutrición humana. De la coor- 


\section{La aceptabilidad de los alimentos: nutrición y placer}

dinación de especialistas de distintas áreas, desde los neurofisiólogos a los tecnólogos de alimentos, pasando por los sicólogos y los especialistas en nutrición, pueden surgir los avances que aseguren al ser humano una alimentación adecuada en las distintas etapas de su vida.

\section{Bibliografía}

AEBI, HE (1981): Food Acceptance and Nutrition: Experiences, intentions and responsabilities. En: Criteria of Food Acceptence. Edts: Solms J y Hall RL. Forster Verlag AG/Forster Publ. Zurich, pp 3-11.

Amerine, M.A., Pangborn, R.M., Roessler, E.B. (1965): Principles of sensory evaluation of foods. Acdemic Press. New York.

Amoore, J.E., Steinle, S. (1991): A graphic history of specific anosmia. En: Chemical Senses. Volume 3. Genetics of perception and communications. Edts: Wysocki ChJ y Kare MR. Marcel Dekker Inc. New York, pp 331-351.

Boelens, M.H. (1991): Spices and condiments. En: Volatiles compounds in foods and beverages. Edt. Maarse H. Marcel Dekker Inc. New York, pp 449-482.

Bоотн, D.A. (1990): Designing products for individual customers. En: Psychological basis of sensory evaluation. Edts. McBride RL y MacFie HJH. Elsevier Appl. Science Londres, 163-193.

BRENNAN, J.G. (1988): Texture perception and measurement. En: Sensory analysis of foods. Edt.:Piggott JR. Elsevier Applied Science. Londres, pp 69-102.

BRown, W.E. (1994): Development of a method to investigate differences in chewing behaviour in humans. I. Use of electromiography in measuring chewing. J. Texture Studies, 25: 1-16.

Cardello, A.V. (1994): Consumers expectations and their role in food acceptance. En: Measurement of food preferences. Edts: MacFie HJH, Thomson DMH. Blakie Academic Prof. Londres, pp 253-297.

Chambers, I.V. E., Smith, E.A. (1991): The uses of qualitative research in product research and development. En: Sensory Science Theory and Applications in Foods. Edts: Lawless HT y Klein BP. Marcel Dekker Inc. New York, pp 395-412.

Costell, E., Durán, L. (2000): Sensory and instrumental measures of viscosity. Selection of instrumental indices. En: Trends in Food Engineering. Edts: Lozano J.E., Añon M C, Parada, E, y Barbosa Canovas G.V. Technomic Publishing CO., Inc. Lancaster, pp. 53-64.

Cowart, B.J., Beauchamp, G.K. (1990): Early development of taste perception. En: Psychological basis of sensory evaluation. Edts. McBride RL y MacFie HJH. Elsevier Appl. Science Londres, 1-17.

Damasio, M.H., Costell, E., Durán, L. (1999): Optimising acceptability of low-sugar strawerry gels segmenting consumers by internal preference mapping. Journal of the Science of Food and Agriculture 79, 626-632.

Delwiche, J. (1996): Are the «basic» tastes?. Trends in Food Science and Technology 7: 411-415.

Durán, L., Costell, E. (1999): Percepción del gusto. Aspectos fisicoquímicos y psicofísicos. Food Science and Technology International, 5: 299-309.

Fischlen, C. (1988): Cuisines and food selection. En: Food Acceptability. Edt.Thomson DMH. Elsevier Appl. Science. Londres, pp 193-206. 


\section{Elvira Costell}

Greenhoff, K., MacFie, H.J.H. (1994): Preference mapping in practice. En: Measurement of food preferences. Edts: MacFie HJH, Thomson DMH. Blakie Academic Prof. Londres, pp 137-166.

Guirao, M. (1981): Los sentidos, bases de la percepción. Editorial Alhambra. Madrid.

Kawanura, Y, Kare, M.R. (1987): Umami. A basic taste. Marcel Dekker. New York.

Kurihara, K., Kashiwayanagi, M., Nomura, T., Yoshis, K., Kumazawa, T. (1989): Chemical stimulus discrimination by specific and nonspecific receptor. Mechanism and their transduction sequences. En Chemical Senses. Volume 1. Receptor Events and Transduction in Taste and Olfaction. Edts: Brand JG, Teeter JH, Cagan RH y Kare MR. Marcel Dekker Inc. New York, pp 55-84.

Kurihara, K. (1987): Recent progress in taste receptor mechanism. En: Umami. A basic taste. Edts. Kawanura Y, Kare MR. Marcel Dekker. New York, pp 3-39.

LAWLESS, H.T. (1991): Bridging the gap between sensory science and product evaluation. En: Sensory Science Theory and Applications in Foods.Edts: Lawless HT y Klein BP. Marcel Dekker Inc. New York, pp 1-36.

Lawless, H.T., HeymanN, H. (1998): Color and appearance. En: Sensory evaluation of foods. International Thomson Publ. Cap. 12, pp 406-429.

MAARSE, H. (1991): Introduction. En: Volatiles compounds in foods and beverages. Edt. Maarse H. Marcel Dekker Inc. New York, pp 1-39.

McBride, R.L., Anderson, N.H. (1990): Integration Psychophysics. En: Psychological basis of sensory evaluation. Edts. McBride RL y MacFie HJH. Elsevier Appl. Science Londres, 93-116.

Meiselman, H.L. (1994): A measurement scheme for developing institutional products. En: Measurement of food preferences. Edts: MacFie HJH, Thomson DMH. Blakie Academic Prof. Londres, pp 1-24.

Mira, F. (1999): El cerebro sintiente. Arbor $\mathrm{n}^{\circ}$ 640, 435-450.

MurPhy, C., GrLmore, M.M. (1990): Effects of aging on sensory functioning: Implications for dietary selection. En: Psychological basis of sensory evaluation. Edts. McBride RL y MacFie HJH. Elsevier Appl. Science Londres, 19-40.

NoRwich, K.H. (1991): Toward the unification of the laws of sensation: Some food for thought. En: Sensory Science Theory and Applications in Foods.Edts: Lawless HT y Klein BP. Marcel Dekker Inc. New York, pp 151-184.

O‘ConELl, R.J. (1991): Specific anosmias: Implications for the physiological mechanism of quality discrimination. En: Sensory Science Theory and Applications in Foods. Edts: Lawless HT y Klein BP. Marcel Dekker Inc. New York, pp 125-150.

Shaw, P.E. (1991): Fruits II. En: Volatiles compounds in foods and beverages. Edt. Maarse H. Marcel Dekker Inc. New York, pp 305-328.

SHEPHERD, R. (1989): Factors influencing food preferences and choice. En: Handbook of the Psychophysiology of Human Eating.Edt. Shepherd R. John Wiley \& Sons Ltd., pp 1-24.

SHEPHERD, R. (1990): Attitudes and beliefs as determinanat of food choice. En: Psychological basis of sensory evaluation. Edts. McBride RL y MacFie HJH. Elsevier Appl. Science Londres, pp 141-162.

STEvens, D.A. (1991): Individual differences in taste and smell. En: Sensory Science Theory and Applications in Foods.Edts: Lawless HT y Klein BP. Marcel Dekker Inc. New York, pp 295-316. 


\section{La aceptabilidad de los alimentos: nutrición y placer}

Stevens, S.S. (1975): Psychophysics: Introduction to its perceptual, neural and social prospects. John Wiley \& Sons. New York.

UNE 87003 (1995). Análisis sensorial. Metodología. Método de investigación de la sensibilidad gustativa. Asociación Española de Normalización y Certificación. Madrid.

VAN Toller, S. (1994): Psychology and psycophysiological measurement of flavor. En: Understanding natural flavors. Edts: Piggott JR, Paterson A. Blakie Academic Prof. Londres, pp 36-59.

Williams, A.A. (1988): Procedures and problems in optimizing sensory and attitudinal characteristics in foods and beverages. En: Food Acceptability. Edt.Thomson DMH. Elsevier Appl. Science. Londres, pp 297-309.

WYSOCKI, Ch.J., BEAUCHAMP, G.K. (1991): Individual differences in human olfaction. En: Chemical Senses. Volume 3. Genetics of perception and communications. Edts: Wysocki ChJ y Kare MR. Marcel Dekker Inc. New York, pp 353-373. 